\title{
MATERIALS FOR THE 21ST CENTURY
}

口 Jessica Wade, Sebastian Wood - DOI: https://doi.org/10.1051/epn/2018502

- Department of Physics, Imperial College London, Prince Consort Road, SW7 2BW

The combined challenges of growing populations, climate change and increasing scarcity of raw materials is driving innovation in materials and design. The motivation to innovate comes from the economic impact of not doing so, as well as consumer demand, with mature customers actively seeking out more environmentally sustainable solutions.

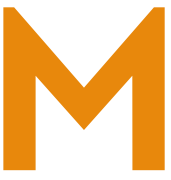

aterials science is at the centre of modern society; essential for scientific and industrial advancement. This article considers the impact of a growing (and developing) global population and climate change on materials design and manufacture. The global population is expected to reach 9.6 billion by 2050 , with an estimated $90 \%$ of people living in cities. It is essential that scientists and urban planners work together to design and protect materials, or this urban growth will result in increased pollution, infrastructural congestion and socioeconomic inequality. In October 2018, the UN Intergovernmental Panel on Climate Change warned we must make 'unprecedented changes' to reach the target of less than $2{ }^{\circ} \mathrm{C}$ of warming. Alongside constructing smarter and more energy efficient homes, new materials will also be required for transportation and healthcare.

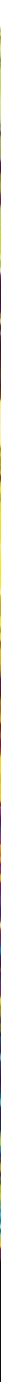


Climate change is poised to become the ultimate design challenge for civil engineers and architects. It will test the way we design, construct, inhabit and maintain the built environment. Not only will the cities of the future have to be less energy and carbon intensive, but they will also need to handle hotter summers, warmer winters, and an increasing occurrence of 'freak' weather conditions, whilst also catering for rapidly growing populations. As well as considering a changing climate in the design of new materials for construction, there is also increasing pressure to reconsider contemporary building materials - many of which perform poorly even today. At the same time, scientists are developing materials for sustainable and renewable energy production, efficient transmission, and storage. Research into new materials can provide low-carbon, low-consumption development solutions. The challenge is providing better performance than existing technologies, with lower environmental costs, at an affordable price.

\section{Energy production}

The stretchability, softness and low-cost processing of organic (carbon-based) materials have attracted considerable attention from researchers across the world. In particular, the class of electrically conducting and semiconducting conjugated polymers offers great promise for a wide range of electronic materials. In contrast to conventional (inorganic) electronic materials, the weak intermolecular bonding results in organic materials being soluble, meaning that they can be printed with relative ease. For almost four decades, they have been used in organic light emitting diodes (OLEDs), photovoltaic cells (OPVs) and transistors. OLEDs were the first organic devices to go to market, and can today be found in highend mobile phones, digital cameras, laptops, televisions and lighting panels. Whilst conventional LEDs still use liquid crystalline filters with backlighting, individual pixels of organic materials emit their own light, resulting in higher energy efficiency, wide viewing angles, vivid colours and exceptional contrast. As of 2018, organic photovoltaics have achieved a remarkable efficiency of $17 \%$ (Figure 1), thanks to the development of new materials and better control over heterojunction structure. This was achieved by combining low and high band-gap materials in a tandem structure.[1] Energy-efficient electrochromic windows demonstrated by the University of Princeton reduce the transmission of sunlight on hot days to keep the rooms inside cool and let heat (near-IR radiation) in on cold days. [2] The smart windows contain transparent solar cells that absorb near-UV light, generating electricity to change the windows colour. Hybrid organic-inorganic perovskite based materials boast extraordinary light absorption and charge-carrier lifetime, and have received significant academic and industry interest. In just a few years, perovskite based solar cell efficiencies have increased from $4 \%$ to $23 \%$, making them competitive with silicon in terms of performance. The design space for next generation materials is enormous, and chemical synthesis is costly and time-consuming. Chemists are now partnering with computer scientists to combine quantum chemistry and artificial intelligence. The virtual molecules are evaluated for their potential as new materials, with the results being fed back into the platform to inform the next generation.

\section{Construction}

Concrete is the most commonly produced and consumed material on earth and makes up $70 \%$ of Europe's infrastructure. It isn't very durable and so maintenance is expensive - an estimated $€ 6$ billion per year - and producing concrete results in up to $12 \%$ of the world's carbon dioxide emissions. In 2015, Jonkers et al. at Delft University, demonstrated Basilisk, a self-healing concrete using limestone-producing bacteria and calcium lactate.[3] The bacteria lie dormant for many years, waking up when the concrete is damaged and water starts to seep through the cracks (Figure 2). When this happens, the bacteria start to feed on calcium lactate, which converts it to insoluble limestone that seals cracks.

\section{Infrastructure}

Air-conditioning units account for $10 \%$ of the global energy consumption and often involve coolants that deplete the ozone layer or contribute to the greenhouse effect. The need for air-conditioning can be reduced by simply painting buildings with white reflective paint to

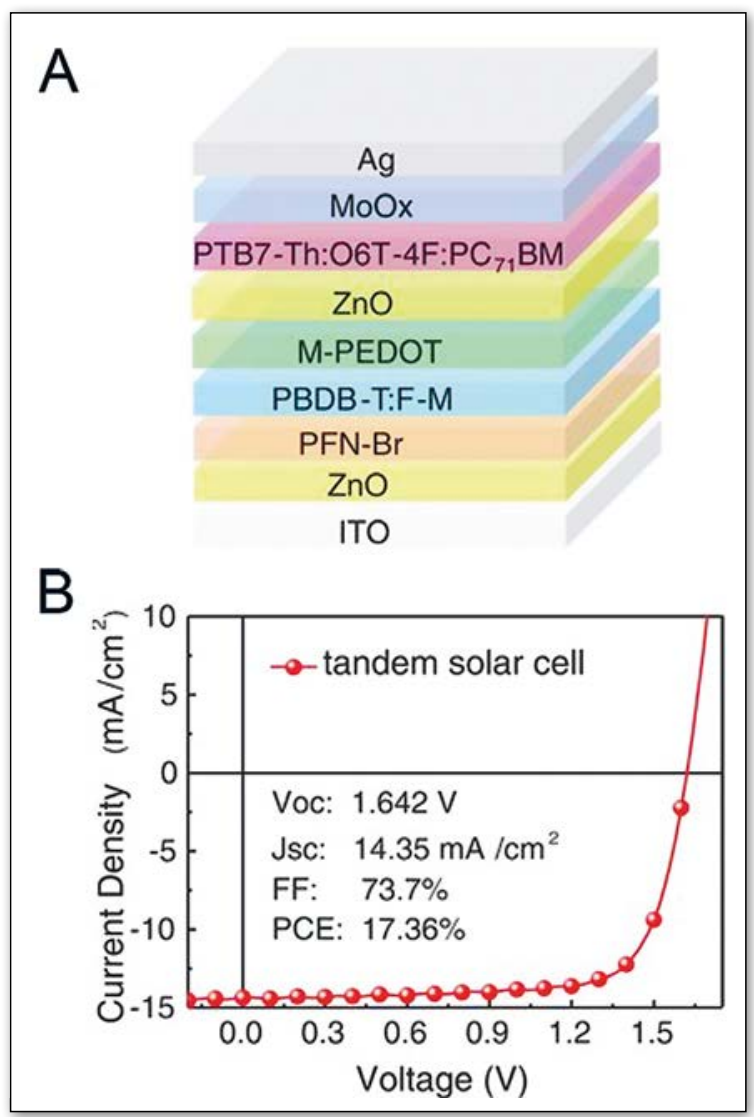

4FIG. 1: A: Device architecture of record-breaking tandem solar cell comprising multiple layers of organic and inorganic materials. B: Current-voltage characteristic of solar cell under 1 Sun illumination showing power conversion efficiency (PCE) of $17.36 \%$. From [1] 


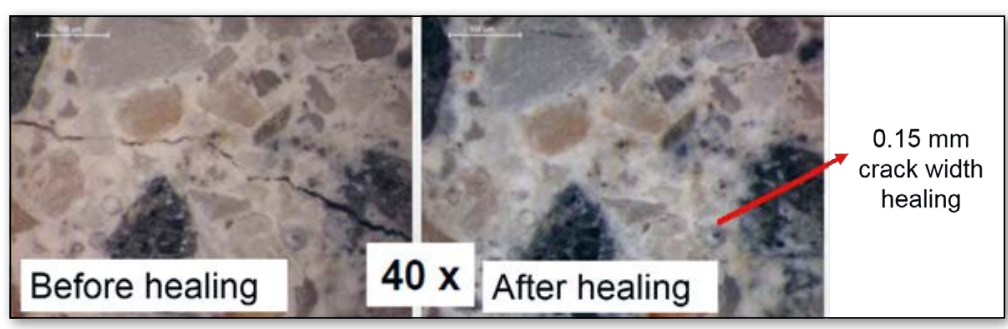

$\triangle F I G$. 2: keep them cool, however this strategy is limited by the Optical micrograph images of selfhealing bacterial concrete sample showing a crack with width $0.15 \mathrm{~mm}$ before (left) and after (right) healing. From https://www. raeng.org.uk/ publications/other/ bioconcrete-a-novelbio-based-material poor broadband reflectiveness of the paint. Researchers at Columbia University have combined a transparent polymer paint with nanoscale air pores, in order to give a white surface that strongly scatters all wavelengths of light (Figure 3).[4] Thin films of a polymer, poly(vinylidene fluoride-co-hexafluoropropene), are prepared from a blend of acetone and water, which creates a series of droplets and microdroplets. As the droplets evaporate, a coating forms that can result in a $6^{\circ} \mathrm{C}$ cooling compared to room temperature. Advances like this seem small in the wider context of global energy use, so it's important to also consider the energy efficiency of whole buildings. The University of Swansea has developed the view of 'buildings as power stations', through their SPECIFIC initiative (Figure 4), combining full-scale technologies such as photovoltaics, thermoelectric generation, lowcost batteries and heat storage. This comprehensive view of building efficiency also encompasses the use of waste heat, which they convert back into useful electricity through tin selenide themoelectric generators. [5]

Transport infrastructure also has a key role to play in sustainable development, and the aerospace sector continues to explore significant advances in materials design. Whilst aluminium once made up $70 \%$ of aeroplanes, titanium, carbon fibre, ceramic-matrix composites and heat-resistant alloys are offering improved efficiency and fuel economy. Ceramic-matrix composites that are reinforced with silicon carbide are low weight and hard, as well as being incredibly thermally and chemically stable. They are one of the fastest growing trends in the aviation industry, with their use in aircraft engines projected to double in the next five years. Carbon fibre is already the main material in the fuselages of the Airbus A350 and the Boeing 787.

\section{Supply and demand}

As globalisation improves living standards around the world, we are at risk of running out of the raw materials that modern life relies on. Rare-earth metals, much coveted for their specific properties, enable high-performance electronic devices with greater energy efficiency. This demand is resulting in the politicisation of mining rare-earths, with prices dependent on trade agreements, scarcity, and political maneuverings, but there is also a high environmental cost to these activities.

\section{Storage}

Lithium-ion (Li-ion) batteries have powered the start of the $21^{\text {st }}$ century, however, we're reaching the limit of their efficiency and they don't age well. Supercapacitors, the energy storage sensation that powers Serbia's e-buses, store their energy in an electric field - free from chemical reactions, they do not suffer from the degradation that plagues Li-ion batteries. On their own, supercapacitors can't store energy for very long. Several energy storage startups have turned to the wonder material of the $21^{\text {st }}$ century: graphene. Carbon-based supercapacitors offer high capacity energy storage and short charging times in battery technology, with researchers trying to identify the perfect nanostructures. Whilst it is hard not to get carried away with all of the promises of lightweight electric supercars, it is important to remember that the production of graphene still requires harsh chemicals or expensive

\footnotetext{
DFIG. 3:

A: Formation of hierarchically porous polymer coating through phase inversion process. B: Micrographs of top surface and crosssection of porous polymer coating. C: Schematic showing high reflectance and thermal emission from coating resulting in cooling effect. From [4]
}

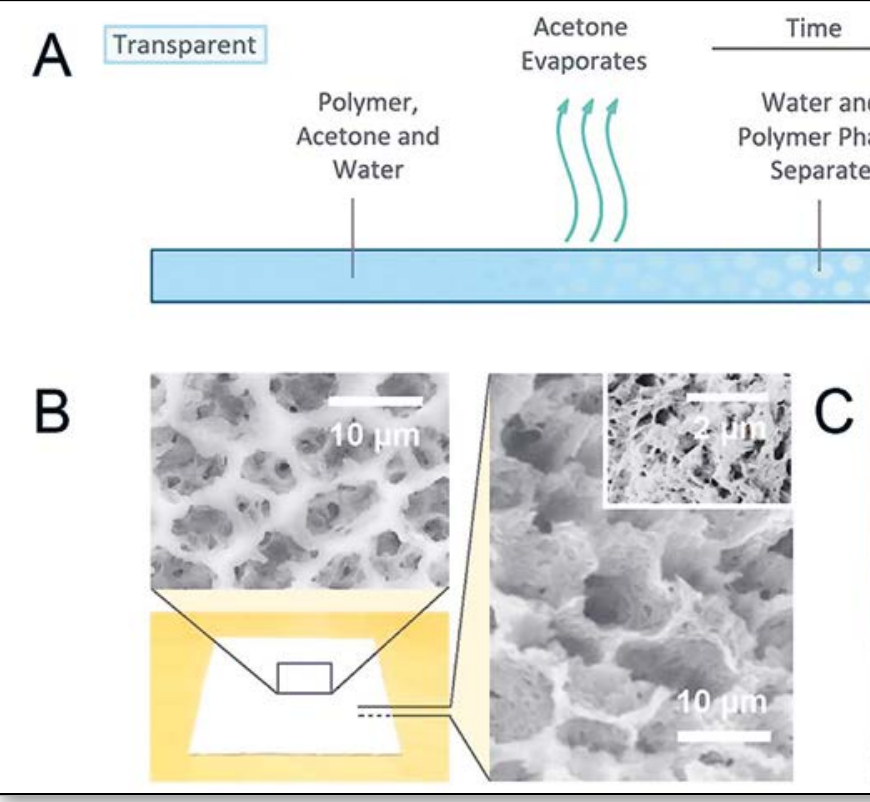

ime

Water

porates

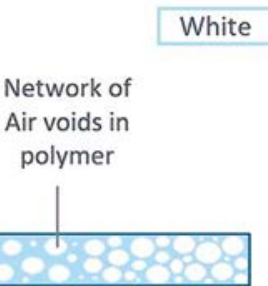

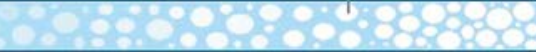

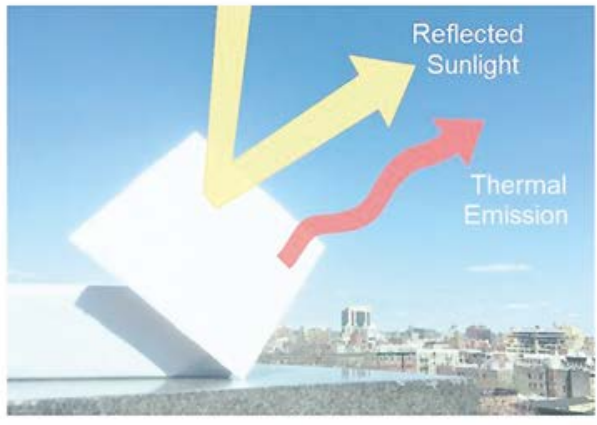


processes, and there are still quality control challenges. The Graphene Flagship project is Europe's largest ever research initiative, which indicates both the perceived benefit of graphene in industry, but also the enormous challenges that must first be addressed.

\section{Future healthcare}

Thanks to the inherent softness of the materials they are made from, organic electronic devices can conform to human tissue or even provide conducting scaffolds between cells. They can transport electrons, holes and ions - which makes them compatible with conventional solid-state electronics as well as biological systems, as a result they are well-suited for applications in biological sensors, implanted electrodes, drug delivery systems and artificial muscles. One of the most versatile conjugated materials for bioelectronics is poly(3,4-ethylenedioxythiophene) (PEDOT), which can be printed from an aqueous dispersion making it compatible with low-cost, stretchable large area devices.

As additive printing becomes more commonplace in manufacture it will revolutionise manufacturing, reducing materials waste and increasing the scope for personalisation. It will shorten supply chains, make work more collaborative and improve repair and maintenance. Manufactures can create innovative structures using layer-by-layer growth, as well as recycling plastic waste for new applications.[6]

\section{Plastics}

We manufacture over 300 million tonnes of plastic a year. Only $14 \%$ of plastic bottles being recycled and, as consumers become more environmentally aware, there is increased concern about the long-term impact of plastic on wildlife. Plastic bans have been announced in Chile, Botswana, Peru and India. In 2018 LEGO partnered with the World Wildlife Foundation to create a range of sustainable bricks made from plant-based materials. But these plant-based plastics are not biodegradable, and their production is still environmentally intensive. Serendipity has played a key role in a number of great scientific breakthroughs, and the accidental discovery, this year, of enzymes that can break down plastic might yet prove to be the key to unlocking the problem of plastic recycling.[7]

\section{Conclusion}

At a recent symposium on printed electronics, a speaker from a large, far-eastern manufacturer of consumer electronics was asked whether they considered recyclability in the development of new products - their answer was an un-nuanced 'no'. Whilst that attitude is by no means universal, one can't help feeling that those organisations which are most sustainability aware are generally smaller and will struggle in a global market with less

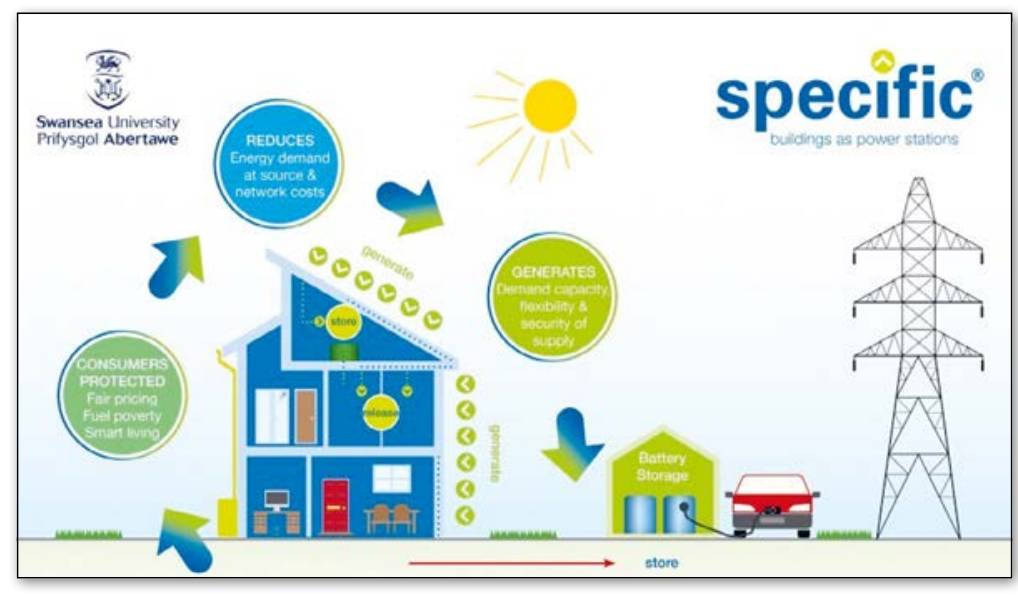

environmentally-driven competitors. This serves as an important reminder that sustainable development is a global issue, and so requires a global solution.

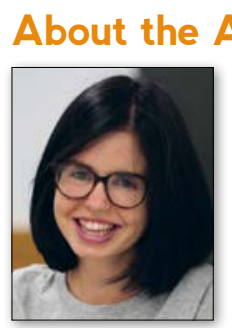

Jessica Wade is a postdoctoral research associate in the Department of Physics and Centre for Plastic Electronics at Imperial College London, creating chiral molecular structures as the active layer for electronic devices. She is involved with several projects to improve diversity within science, working closely with the Institute of Physics and Women's Engineering Society.

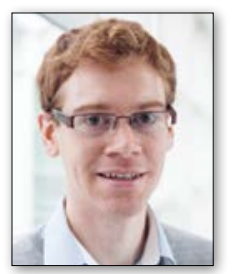

Sebastian Wood is a Visiting Researcher at Imperial College London and a Senior Research Scientist based at the National Physical Laboratory (NPL) working in the field of printed and emerging electronic technologies. NPL is positioned at the interface between government, academia, and industry with the role of supporting UK industry and quality of life through measurement science and standardisation.

\section{References}

[1] L. Meng, Y. Zhang, X. Wan, C. Li, X. Zhang, Y.Wang, X. Ke, Z. Xiao, L. Ding, R. Xia, H. L. Yip, Y. Cao, Y. Chen, Science 361, 1094 (2018)

[2] N. C. Davy, M. Sezen-Edmonds, J. Gao, X. Lin, A. Liu, N. Yao, A. Kahn, Y.-L. Loo, Nat. Energy 2, 17104 (2017)

[3] H. M. Jonkers, A. Thijssen, G. Muyzer, O. Copuroglu, E. Schlangen, Ecol. Eng. 36, 230 (2010)

[4] J. Mandal, Y. Fu, A. Overvig, M. Jia, K. Sun, N. Shi, H. Zhou, X. Xiao, N. Yu, Y. Yang, Science (2018), doi:10.1126/science.aat9513.

[5] M. R. Burton, T. Liu, J. McGettrick, S. Mehraban, J. Baker, A. Pockett, T. Watson, O. Fenwick, M. J. Carnie, Adv. Mater. 2018, 30, DOI 10.1002/adma.201801357.

[6] M. Despeisse, S. Ford, IFIP Adv. Inf. Commun. Technol., 129 (2015)

[7] H. P. Austin, M. D. Allen, B. S. Donohoe, N. A. Rorrer, F. L. Kearns, R. L. Silveira, B. C. Pollard, G. Dominick, R. Duman, K. El Omari, V. Mykhaylyk, A. Wagner, W. E. Michener, A. Amore, M. S. Skaf, M. F. Crowley, A. W. Thorne, C. W. Johnson, H. L. Woodcock, J. E. McGeehan, G. T. Beckham, Proc. Natl. Acad. Sci. (2018), 201718804 $\triangle$ FIG. 4: Schematic illustrating the 'buildings as power stations' concept where a residential building generates, stores, and releases energy. From specific.eu.com 